\title{
Bubble CPAP to support preterm infants in rural Rwanda: a retrospective cohort study
}

Evrard Nahimana ${ }^{1,5^{*}}$, Masudi Ngendahayo ${ }^{2}$, Hema Magge ${ }^{1,3,4}$, Jackline Odhiambo ${ }^{1}$, Cheryl L. Amoroso ${ }^{1}$, Ernest Muhirwa ${ }^{1}$, Jean Nepo Uwilingiyemungu ${ }^{2}$, Fulgence Nkikabahizi ${ }^{2}$, Regis Habimana ${ }^{2}$ and Bethany L. Hedt-Gauthier ${ }^{1,5}$

\begin{abstract}
Background: Complications from premature birth contribute to $35 \%$ of neonatal deaths globally; therefore, efforts to improve clinical outcomes of preterm (PT) infants are imperative. Bubble continuous positive airway pressure (bCPAP) is a low-cost, effective way to improve the respiratory status of preterm and very low birth weight (VLBW) infants. However, bCPAP remains largely inaccessible in resource-limited settings, and information on the scale-up of this technology in rural health facilities is limited. This paper describes health providers' adherence to bCPAP protocols for PTNLBW infants and clinical outcomes in rural Rwanda.

Methods: This retrospective chart review included all newborns admitted to neonatal units in three rural hospitals in Rwanda between February 1st and October 31st, 2013. Analysis was restricted to PTNLBW infants. bCPAP eligibility, identification of bCPAP eligibility and complications were assessed. Final outcome was assessed overall and by bCPAP initiation status.

Results: There were 136 PTNLBW infants. For the 135 whose bCPAP eligibility could be determined, $83(61.5 \%)$ were bCPAP-eligible. Of bCPAP-eligible infants, 49 (59.0 \%) were correctly identified by health providers and 43 (51.8\%) were correctly initiated on bCPAP. For the 52 infants who were not bCPAP-eligible, $45(86.5 \%)$ were correctly identified as not bCPAP-eligible, and 46 (88.5\%) did not receive bCPAP. Overall, 90 (66.2\%) infants survived to discharge, 35 (25.7\%) died, 3 (2.2\%) were referred for tertiary care and 8 (5.9\%) had unknown outcomes. Among the bCPAP eligible infants, the survival rates were $41.8 \%$ (18 of 43) for those in whom the procedure was initiated and $56.5 \%$ (13 of 23) for those in whom it was not initiated. No complications of bCPAP were reported.

Conclusion: While the use of bCPAP in this rural setting appears feasible, correct identification of eligible newborns was a challenge. Mentorship and refresher trainings may improve guideline adherence, particularly given high rates of staff turnover. Future research should explore implementation challenges and assess the impact of bCPAP on long-term outcomes.
\end{abstract}

Keywords: bCPAP, Very low birth weight, Preterm, Premature, Respiratory distress, CPAP, Rwanda, Africa

\section{Background}

Over 2.9 million neonatal deaths occur every year, representing $44 \%$ of all under five deaths [1-3]. In Rwanda, despite a rapid decline in under-five mortality, the number of deaths in the neonatal period remains high $(27 / 1000$ live births) with little change over the past 10 years $[4,5]$.

\footnotetext{
* Correspondence: evnahimana@gmail.com

'Partners In Health/Inshuti Mu Buzima, Rwinkwavu, Rwanda

${ }^{5}$ Department of Global Health and Social Medicine, Harvard Medical School, 02115 Boston, MA, USA

Full list of author information is available at the end of the article
}

Major causes of neonatal deaths include preterm birth, birth asphyxia and infections. Recently, complications related to prematurity have surpassed pneumonia and diarrheal diseases as the number one cause death in children, and account for $35 \%$ of all neonatal deaths [1-3, 6-8]. Hospital-based interventions targeting these causes are needed to reduce neonatal mortality, particularly in low and middle income countries [9-11].

The implementation of hospital-based interventions is challenging in resource limited settings. Specifically, intensive care unit technology for respiratory distress, such

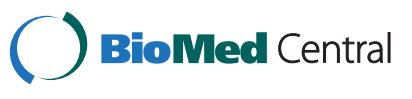

(C) 2015 Nahimana et al. Open Access This article is distributed under the terms of the Creative Commons Attribution 4.0 International License (http://creativecommons.org/licenses/by/4.0/), which permits unrestricted use, distribution, and reproduction in any medium, provided you give appropriate credit to the original author(s) and the source, provide a link to the Creative Commons license, and indicate if changes were made. The Creative Commons Public Domain Dedication waiver (http://creativecommons.org/publicdomain/zero/1.0/) applies to the data made available in this article, unless otherwise stated. 
as a mechanical ventilation, is often not available due to high costs, maintenance demands and the need for highly trained staff. However, continuous positive airway pressure (CPAP) has been demonstrated to be a simple, low-cost and effective alternative to improve the respiratory status of preterm infants with respiratory distress syndrome [12, 13], and decrease the need for conventional mechanical ventilators $[12,14]$. CPAP helps keep the respiratory tract and lungs open, promotes comfortable breathing, improves oxygen levels and decreases apnea in premature infants. Bubble CPAP (bCPAP) is the least expensive and least complicated CPAP option, making this the preferred technology in resource-limited settings $[15,16]$.

To date, few studies have been conducted to show the impact and feasibility of bCPAP in areas with limited resources. These studies, most of which were conducted in teaching and/or urban hospitals, have shown that bCPAP can reduce the need for mechanical ventilation and can be applied by nurses after a short on-the-job training on the protocol and equipment [12, 17]. However, little research has been done on the use of bCPAP in rural resourcelimited settings and hospitals without pediatric specialists.

In January 2013, the Rwandan Ministry of Health $(\mathrm{MOH})$, in collaboration with Partners In Health (PIH), introduced a bCPAP program integrated into broader neonatal care services for newborns with respiratory distress in three rural district hospitals (Butaro, Kirehe and Rwinkwavu District Hospitals). Nurses and general practitioners working in the neonatal units in these hospitals with a background in neonatal care services received intensive training on advanced neonatal care, focusing on the bCPAP protocol, safe assembly, maintenance and trouble-shooting of different issues related to bCPAP use. The training was supplemented by ongoing clinical mentorship and intermittent refresher trainings led by $\mathrm{PIH}$ and local MOH bCPAP champions.

The objectives of this study are to describe the provider adherence to bCPAP protocol for preterm and very low birth weight (PT/VLBW) infants and to describe the outcomes of these infants at the three district hospitals. The ultimate goal is to better understand the use of bCPAP in rural resource-limited settings in order to improve the quality of bCPAP implementation and inform the scale-up of this technology in similar settings.

\section{Methods}

This retrospective cohort study included infants receiving care at neonatal units at Rwinkwavu, Kirehe and Butaro District Hospitals from February 1, 2013 to October 31, 2013. The catchment area included 865,000 people and care at the hospital was obtained after referral from one of the 41 health centers within the districts. These three hospitals were selected for the study as they were the only rural district hospitals providing basic neonatal care using
bCPAP in Rwanda in 2013. A team of nurses and general practitioners worked permanently in these units providing care to an average of 25 infants every month in each hospital. Infants who needed intensive neonatal care, including mechanical ventilators, were referred to tertiary hospitals in Kigali city (the capital of Rwanda). Following the training on implementation of bCPAP, Rwinkwavu and Kirehe District Hospitals benefited from fairly consistent mentorship from PIH pediatric specialists during the study period while Butaro hospital had more intermittent specialist presence.

Respiratory assessment to determine the need for bCPAP is based on physical examination (such as grunting, nasal flaring and chest retraction) and vital signs (including respiratory rate and/or oxygen saturation). In addition, the etiology of respiratory symptoms and the natural history of that diagnosis are considered. Once the overall assessment is complete, the degree of respiratory distress is categorized as mild, moderate or severe. Moderate to severe signs include moderate to severe grunting, flaring, retractions and respiratory rate $>70$ or $<30$ and/or oxygen saturation $<90 \%$ (The oxygen saturation was measured using pulse oximeter). Based on the bCPAP protocol used in the three district hospitals, any newborn with a moderate to severe respiratory distress should have been initiated on bCPAP (Fig. 1). Furthermore, preterm (gestational age (GA) $<33$ weeks) or very low birth weight $(<1500 \mathrm{~g})$ infants with any degree of respiratory distress (mild, moderate or severe) should have been initiated on bCPAP. Preterm infants with significant apnea and bradycardia of prematurity were also eligible.

Our study population included all PT/VLBW infants admitted in neonatology units at the three hospitals. All term and near term infants (GA $\geq 33$ weeks and/or birth weight $\geq 1500 \mathrm{~g}$ ) were excluded as the severity of their respiratory distress was not captured in the patient charts and therefore eligibility for bCPAP could not be ascertained. For infants included in the study, we added a category of unknown to indicate missing data. The following information was extracted from the patient charts and registers in the neonatology and maternity unit: place of birth, birth weight, gestational age, respiratory rate, oxygen saturation, presence of physical signs of respiratory distress (grunting, chest retraction, nasal flaring), bCPAP recommendation and initiation, final disposition (recovered, referred or died) and presence of bCPAP complications (skin injury, pneumothorax, abdominal distention). We categorized PT/VLBW infants with at least one sign of respiratory distress as $\triangle C P A P$ eligible and those without any sign of respiratory distress as $b C P A P$ ineligible. Data was extracted into a standard data collection form, and a file linking the study ID to the mother and neonate ID was kept separately during the data collection and destroyed 


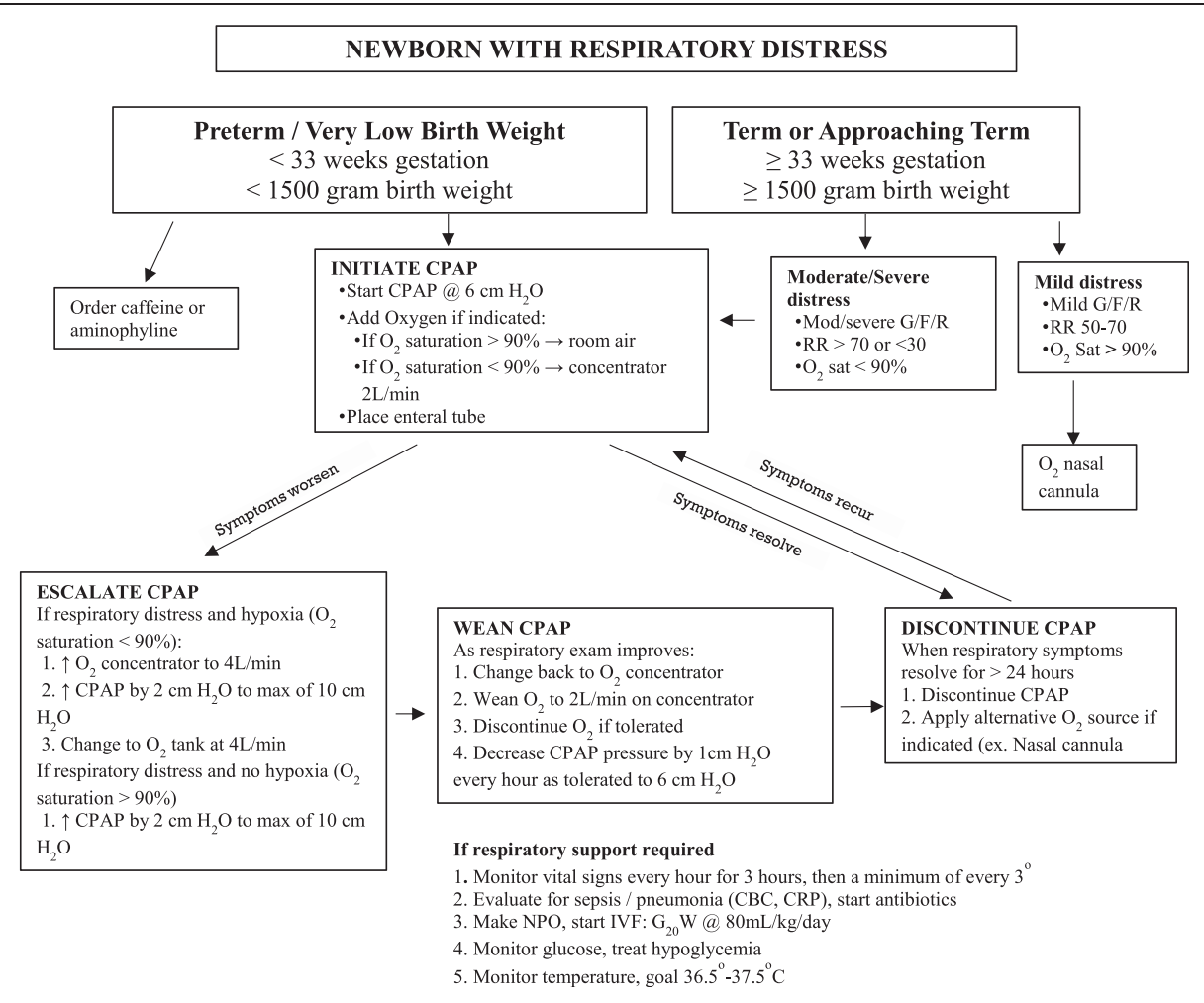

Fig. 1 CPAP indication and implementation for newborns with respiratory distress based on the Rwanda CPAP protocol 2013

after data validation. We analyzed data using Stata 12.1 (College Station, TX: StataCorp LP). We used descriptive statistics reporting number and percent of infant characteristics, infants identified as eligible for bCPAP, infants for whom bCPAP was initiated and clinical outcomes based on CPAP eligibility. We also used median and interquartile range for the duration of stay in the hospital.

The study received technical and ethical approvals from Rwanda institutional review boards: The Inshuti $\mathrm{Mu}$ Buzima Research Committee (IMBRC), the National Health Research Committee (NHRC) and the Rwanda National Ethics Committee (RNEC). As the study used deidentified routinely corrected data, the consent for parents was waived. STROBE (STrengthening the Reporting of OBservational studies in Epidemiology) guidelines were also followed for this study.

\section{Results}

During the study period, 862 infants were admitted in the three hospitals. Of these, 136 (16\%) were identified as PT/ VLBW and included in the analysis (Table 1). Of the 136 infants, $75.7 \%(n=103)$ were VLBW and $57.4 \%(n=78)$ were preterm. Most of the PT/VLBW infants $(n=117$, $86 \%)$ were born at a health facility, either hospital or health center. The median number of days of stay at the hospital was 19 with an interquartile range of 6-32 days. In assessing the presence of respiratory distress symptoms among PT/VLBW infants, $61.0 \%(n=83)$ showed at least one sign of respiratory distress (Table 2). Many of the infants (50.7\%, $n=69)$ had low oxygen saturation $\left(\mathrm{SpO}_{2}<90 \%\right)$ and 38 infants (28.4\%) had chest retraction. In some cases, the clinicians only mentioned infants in respiratory distress without specifying the physical symptoms. One infant did not have documentation of the presence or absence of respiratory distress and thus, bCPAP eligibility could not be determined.

Of the 135 PT/VLBW infants whose bCPAP eligibility could be determined, $61.5 \%(n=83)$ were bCPAP-eligible of which $59.0 \%(n=49)$ were correctly identified by health providers and for $51.8 \%(n=43)$ bCPAP was initiated. Twenty-three bCPAP-eligible infants (27.7 \%) had no indication of being identified as bCPAP eligible or of being initiated on bCPAP. Information around identification was missing for $13.3 \%(n=11)$ of infants who were eligible (Table 3). For the 52 infants who were not bCPAP-eligible, 45 (86.5\%) were correctly identified as not bCPAP-eligible and $46(88.5 \%)$ did not receive bCPAP.

Overall, among the 136 PT/VLBW admitted, 90 (66.2 \%) infants survived to discharge, 35 (25.7\%) died and 3 $(2.2 \%)$ were referred for tertiary care. Outcome information was missing for 8 (5.9 \%) infants. For the 43 infants who were bCPAP-eligible and for whom bCPAP was initiated, $41.9 \%(n=18)$ recovered and $48.8 \%(n=21)$ died 
Table 1 Characteristics of infants admitted to the neonatology unit in three district hospitals in Rwanda

\begin{tabular}{|c|c|c|c|c|}
\hline \multirow[t]{2}{*}{ Population characteristics } & \multicolumn{2}{|c|}{$\begin{array}{l}\text { Preterm or very low birth weight infants } \\
N=136\end{array}$} & \multicolumn{2}{|c|}{$\begin{array}{l}\text { Term and near term infants who are not very low birth weight } \\
N=726\end{array}$} \\
\hline & $\bar{n}$ & $\%$ & $\bar{n}$ & $\%$ \\
\hline \multicolumn{5}{|l|}{ Place of birth } \\
\hline Hospital & 66 & 48.5 & 419 & 57.7 \\
\hline Health center & 51 & 37.5 & 255 & 35.1 \\
\hline Home & 14 & 10.3 & 32 & 4.4 \\
\hline Unknown & 5 & 3.7 & 20 & 2.8 \\
\hline \multicolumn{5}{|l|}{ Birth weight } \\
\hline Very low birth weight $(<1500 \mathrm{~g})$ & 103 & 75.7 & & \\
\hline Low birth weight (1500-2499 g) & 31 & 22.8 & 224 & 30.9 \\
\hline Normal birth weight (>=2500 g) & 2 & 1.5 & 437 & 60.1 \\
\hline Unknown & 0 & 0 & 65 & 9.0 \\
\hline \multicolumn{5}{|l|}{ Gestation age at birth } \\
\hline$<33$ weeks & 78 & 57.4 & & \\
\hline 33-36 weeks & 19 & 14.0 & 71 & 9.8 \\
\hline$=37$ weeks & 9 & 6.6 & 548 & 75.5 \\
\hline Unknown & 30 & 22.1 & 107 & 14.7 \\
\hline Duration of stay in the hospital & $N=128$ & & $N=705$ & \\
\hline Median (IQR) & $19(6-32)$ & & $7(3-10)$ & \\
\hline 0-7 days & 34 & 25.0 & 413 & 56.9 \\
\hline 8-14 days & 18 & 13.2 & 140 & 19.3 \\
\hline $15-30$ days & 34 & 25.0 & 52 & 7.2 \\
\hline >30 days & 29 & 21.3 & 33 & 4.5 \\
\hline Unknown & 21 & 15.4 & 88 & 12.1 \\
\hline
\end{tabular}

(Table 4). Of the 23 bCPAP-eligible infants for whom bCPAP was not initiated, $56.5 \%(n=13)$ recovered, $39.1 \%$ $(n=9)$ died and information about the outcome was missing for $4.4 \%(n=1)$. Outcome information was missing for 1 (2.3\%) infant. A large proportion of infants who were CPAP ineligible recovered whether bCPAP was initiated (100\%, 2 out 2 ) or not initiated (93.5\%, 43 out of 46$)$. For infants who did receive bCPAP, no complications such as skin injury, pneumothorax or abdominal distention were reported.

\section{Discussion}

In this study, we assessed the implementation of bCPAP with PT/VLBW infants at three district hospitals in rural Rwanda and found the intervention feasible in a resource-limited rural setting. Over the nine-month period, 45 infants were initiated on bCPAP, demonstrating that bCPAP - an evidence-based intervention to improve survival or PT/VLBW infants - is filling a medical care need for neonates. However, only $52 \%$ of bCPAP-eligible infants received bCPAP, suggesting ongoing gaps in correct identification and initiation of eligible infants. We suspect that this low sensitivity might be a result of turnover of nurses and doctors and could be improved with increased onsite mentorship and refresher trainings, particularly to identify early and mild signs of distress promptly for immediate CPAP initiation to gain the full benefit of the intervention. Qualitative research to assess and understand the barriers to implementation experienced by nurses and doctors is also advised.

Conversely, $88.5 \%$ of bCPAP ineligible infants were not initiated, indicating that clinicians are not exposing

Table 2 Evidence of respiratory distress among preterm (<33 weeks) or very low birth weight $(<1500 \mathrm{~g})$ infants

\begin{tabular}{lll}
\hline Sign of respiratory distress $(N=136)$ & \multicolumn{2}{l}{ Infants with symptoms } \\
\cline { 2 - 3 } & $\mathrm{n}$ & $\%$ \\
\hline $\mathrm{SpO}_{2}<90 \%$ & 69 & 50.7 \\
Grunting $(N=134)$ & 17 & 12.7 \\
Chest retraction $(N=134)$ & 38 & 28.4 \\
Nasal flaring $(N=134)$ & 15 & 11.2 \\
Respiration rate $<30$ or $>70$ & 24 & 17.7 \\
At least one sign of respiratory distress & 83 & 61.5 \\
$(N=135)$ & & \\
\hline
\end{tabular}


Table 3 bCPAP identification and initiation for preterm ( $<33$ weeks) or very low birth weight $(<1500)$ infants

\begin{tabular}{|c|c|c|c|c|c|c|}
\hline & \multicolumn{2}{|c|}{$\begin{array}{l}\text { bCPAP eligible } \\
N=83\end{array}$} & \multicolumn{2}{|c|}{$\begin{array}{l}\text { bCPAP Not eligible } \\
N=52\end{array}$} & \multicolumn{2}{|c|}{$\begin{array}{l}\text { Total } \\
N=135^{\mathrm{a}}\end{array}$} \\
\hline & $\bar{n}$ & $\%$ & $n$ & $\%$ & $\bar{n}$ & $\%$ \\
\hline \multicolumn{7}{|c|}{ Identified as bCPAP-Eligible } \\
\hline Yes & 49 & 59.0 & 3 & 5.8 & 52 & 38.5 \\
\hline No & 23 & 27.7 & 45 & 86.5 & 68 & 50.4 \\
\hline Unknown & 11 & 13.3 & 4 & 7.7 & 15 & 11.1 \\
\hline \multicolumn{7}{|c|}{ bCPAP Initiated } \\
\hline Yes & 43 & 51.8 & 2 & 3.9 & 36 & 33.3 \\
\hline No & 23 & 27.7 & 46 & 88.5 & 60 & 51.3 \\
\hline Unknown & 17 & 20.5 & 4 & 7.7 & 21 & 17.9 \\
\hline
\end{tabular}

OOne infant's eligibility could not be determined

ineligible infants to possible bCPAP side effects and conserving the machines for the infants most in need. Only two of the bCPAP initiated infants were bCPAP ineligible according to medical file documentation, an improvement over a study in Malawi where of the 11 neonates treated with bCPAP, six did not meet initiation criteria [16].

A quarter of infants included in this study died before discharge from the hospital. This mortality rate is similar to outcomes of PT/VLBW infants in similar settings in sub-Saharan Africa [18-20]. The highest rate of death in this study, nearly $49 \%$, occurred in infants eligible for CPAP who died after initiation. Given the low sensitivity of CPAP initiation, we suspect that this group had a higher severity of respiratory distress and other comorbidities compared to infants who were not initiated on CPAP. We were unable to accurately assess the severity of respiratory distress among those who were eligible but not initiated on CPAP; however, we suspect that they were likely to be less severely ill. In addition, our study was conducted in rural hospitals without full-time pediatric specialists on staff; however, similarly high mortality rates among bCPAP initiated infants have been reported in studies conducted in teaching hospitals with more specialized staff $[15-17,21]$.
There are several limitations to consider for this study. This study is based entirely on routinely collected data available in the patient file. While we cannot verify the accuracy of diagnosis, we believe the information provided by clinicians is reliable because of their clinical background and expertise. For some cases, however, there was limited documentation from clinicians especially on the severity of respiratory distress. Our study excluded term and near-term infants whose bCPAP eligibility depended on the severity of respiratory distress, which was difficult to capture in patients records. Furthermore, we were unable to assess the degree of distress among eligible infants whom were not provided bCPAP to assess for possible selection bias. In a few cases for the PT/VLBW infants, it was difficult to determine whether the infant was identified for bCPAP or initiated on bCPAP. To improve documentation and resulting quality improvement, we recommend the revision of the neonatology patient chart and onsite training/supervision. Despite these challenges, we believe these results are informative as they represent the first assessment of bCPAP implementation in rural Rwanda and thus provide a basis for informing better service delivery and bCPAP scale-up in similar settings.

Table 4 Clinical Outcomes for Preterm ( $<33$ weeks) or very low birth weight infants $(<1500 \mathrm{~g})$ with and without bCPAP intervention

\begin{tabular}{|c|c|c|c|c|c|c|c|c|}
\hline & \multicolumn{2}{|c|}{$\begin{array}{l}\text { Recovered/ Discharged } \\
N=89\end{array}$} & \multicolumn{2}{|c|}{$\begin{array}{l}\text { Died } \\
N=35\end{array}$} & \multicolumn{2}{|c|}{$\begin{array}{l}\text { Transferred for care } \\
N=3\end{array}$} & \multicolumn{2}{|c|}{$\begin{array}{l}\text { Outcome unknown } \\
N=8\end{array}$} \\
\hline & $n$ & $\%$ & $\mathrm{n}$ & $\%$ & $n$ & $\%$ & $n$ & $\%$ \\
\hline \multicolumn{9}{|l|}{ Eligible } \\
\hline bCPAP Initiated $(N=43)$ & 18 & 41.8 & 21 & 48.8 & 3 & 6.9 & 1 & 2.3 \\
\hline bCPAP Not Initiated $(N=23)$ & 13 & 56.5 & 9 & 39.1 & 0 & 0 & 1 & 4.4 \\
\hline bCPAP Initiation Unknown $(N=17)$ & 10 & 58.8 & 4 & 23.5 & 0 & 0 & 3 & 17.7 \\
\hline \multicolumn{9}{|l|}{ Not eligible } \\
\hline bCPAP Initiated $(N=2)$ & 2 & 100 & 0 & 0 & 0 & 0 & 0 & 0 \\
\hline bCPAP Not Initiated $(N=46)$ & 43 & 93.5 & 0 & 0 & 0 & 0 & 3 & 6.5 \\
\hline bCPAP Initiation Unknown $(N=4)$ & 3 & 75.0 & 1 & 25.0 & 0 & 0 & 0 & 0 \\
\hline
\end{tabular}




\section{Conclusion}

To our knowledge, this is the first study of implementation of bCPAP in rural district hospitals in sub-Saharan Africa. We found that bCPAP is a feasible way to support infants with respiratory distress in resource-limited settings. While the introduction and use of bCPAP in this setting appears promising, there remain challenges in terms of guideline adherence. We believe that providing more intense mentorship and refresher trainings can improve guideline adherence, particularly given the high rates of staff turnover. We also recommend the adaption of clinical charts to facilitate clinical determination of degree of respiratory distress and consequent decision-making. Future qualitative and prospective research is needed to determine challenges encountered by clinicians in using bCPAP as well as delineate the reasons for high mortality among infants put on CPAP. Finally and critically, more research is needed to assess the impact of bCPAP on long-term survival and outcomes for PT/VLBW infants.

\section{Abbreviations}

BCPAP: Bubble continuous positive airway pressure; CPAP: Continuous positive airway pressure; GA: Gestation age; ID: Identification; IMB: Inshuti Mu Buzima; MOH: Ministry of Health; PIH: Partners In Health; PT: Preterm; $\mathrm{SpO}_{2}$ : Oxygen saturation; STROBE: STrengthening the Reporting of OBservational studies in Epidemiology; VLBW: Very low birth weight.

\section{Competing interests}

The authors declare that they have no competing interests.

\section{Authors' contributions}

EN and MN led the study design, data collection oversight, data analysis and interpretation, and manuscript preparation. HM provided inputs on study design, data analysis and interpretation, and manuscript preparation. JO supported data collection oversight, data analysis and interpretation, and manuscript preparation. CA supported determination of study design, data analysis, interpretation, manuscript preparation and team mentorship. EM, JNU, FN and RH supported study design, data interpretation, and manuscript preparation. BHG supervised the research process and provided inputs in study design, data analysis, interpretation, manuscript preparation and mentorship. All authors read and approved the final manuscript.

\author{
Authors' information \\ EN: MD, District Clinical Director, Partners In Health/Inshuti Mu Buzima, \\ Rwanda. \\ MN: MD, Head of Pediatric Department, Kirehe District Hospital, Ministry of \\ Health/ Rwanda. \\ HM: MD, Director of Pediatrics, Partners In Health/Inshuti Mu Buzima, \\ Rwanda. \\ JO: BA, Research Fellow, Partners In Health/Inshuti Mu Buzima, Rwanda. \\ $\mathrm{CA}: \mathrm{MPH}$, Director of Research, Monitoring and Evaluation and Health \\ Information Systems, Partners In Health/Inshuti Mu Buzima, Rwanda. \\ EM: Monitoring and evaluation coordinator, Partners In Health/Inshuti Mu \\ Buzima, Rwanda. \\ JNU: MD, Director of Kirehe Hospital, Ministry of Health/Rwanda. \\ FN: MD, Director of Rwinkwavu Hospital, Ministry of Health/Rwanda. \\ RH: MD, Head of Pediatric Department, Butaro Hospital, Ministry of Health/ \\ Rwanda. \\ BHG: PhD, Research Advisor, Partners In Health/Inshuti Mu Buzima, Rwanda.
}

\section{Acknowledgements}

We acknowledge Partners In Health/Inshuti Mu Buzima and the IMB Innovation Grants for the support of this work. This study was developed under the Partners In Health/Inshuti Mu Buzima Intermediate Operational Research Training Program, developed and facilitated by Bethany Hedt-Gauthier and Cheryl Amoroso. Authors also thank $\mathrm{PIH}$ and the $\mathrm{MOH}$ for providing support for the implementation of bCPAP at the three districts. We express our gratitude to the data collectors Lisa Munyana, Michel Nshimiyimana, Lievin Bayahunde and Liliose Mukantaganzwa.

\section{Author details}

${ }^{1}$ Partners In Health/Inshuti Mu Buzima, Rwinkwavu, Rwanda. ${ }^{2}$ Ministry of Health, Kigali, Rwanda. ${ }^{3}$ Division of General Pediatrics, Boston Children's Hospital, Boston, USA. ${ }^{4}$ Division of Global Health Equity, Brigham and Women's Hospital, Boston, USA. ${ }^{5}$ Department of Global Health and Social Medicine, Harvard Medical School, 02115 Boston, MA, USA.

Received: 28 January 2015 Accepted: 11 September 2015

Published online: 24 September 2015

\section{References}

1. United Nations Children's Fund (UNICEF), World Health Organization (WHO), The World Bank. Levels and trends in child mortality: report 2013. New York: UNICEF; 2013

2. Lawn JE, Blencowe H, Oza S, You D, Lee AC, Waiswa P, et al. Every Newborn: progress, priorities and potential beyond survival. Lancet. 2014;384(9938):189-205.

3. Liu L, Oza S, Hogan D, Perin J, Rudan I, Lawn JE, et al. Global, regional, and national causes of child mortality in 2000-13, with projections to inform post-2015 priorities: an updated systematic analysis. Lancet. 2014. http:// dx.doi.org/10.1016/S0140-6736(14)61698-6.

4. Farmer PE, Nutt CT, Wagner CM, Sekabaraga C, Nuthulaganti T, Weigel JL, Farmer DB, Habinshuti A, Mugeni SD, Karasi JC, Drobac PC. Reduced premature mortality in Rwanda: lessons from success. Br Med J. 2013;346. http://dx.doi.org/10.1136/bmj.f65.

5. Rwanda Demographic and Health Survey 2010. http://www.statistics.gov.rw/ publications/demographic-and-health-survey-2010-final-report.

6. Blencowe H, Cousens S, Chou D, Oestergaard M, Say L, Molle AB, et al. Born Too Soon: the global epidemiology of 15 million preterm births. Reprod Health. 2013;10 Suppl 1:S2.

7. Cooper PA. The challenge of reducing neonatal mortality in low-and middle-income countries. Pediatrics. 2014;133(1):4-6.

8. Black RE, Cousens S, Johnson HL, Lawn JE, Rudan I, Bassani DG, et al. Global, regional, and national causes of child mortality in 2008: a systematic analysis. Lancet. 2010;375:1969-87. http://dx.doi.org/10.1016/S0140-6736(10)60549-1.

9. Bhutta ZA, Das JK, Bahl R, Lawn JE, Salam RA, Paul VK, et al. Can available interventions end preventable deaths in mothers, newborn babies, and stillbirths, and at what cost? Lancet. 2014;84(9940):347-70. http://dx.doi.org/ 10.1016/S0140-6736(14)60792-3

10. Lawn JE, Kinney MV, Belizan JM, Mason EM, McDougall L, Larson J, et al. Born Too Soon: accelerating action for prevention and care of 15 million newborns born too soon. Reprod Health. 2013;10 Suppl 1:S6. doi:10.1186/ 1742-4755-10-S1-S6.

11. Dickson KE, Simen-Kapeu A, Kinney MV, Huicho L, Vesel L, Lackritz E, et al. Every Newborn: health-systems bottlenecks and strategies to accelerate scale-up in countries. Lancet. 2014;384(9941):438-54. http://dx.doi.org/ 10.1016/S0140-6736(14)60582-1.

12. Martin S, Duke T, Davis P. Efficacy and safety of bubble CPAP in neonatal care in low and middle income countries: a systematic review. Arch Dis Child . 2014;99(6):F495-504.

13. Brown J, Machen H, Kawaza K, Mwanza Z, Iniguez S, Lang H, et al. A high-value, low-cost bubble continuous positive airway pressure in low-resource settings: technical assessment and initial case reports. PLoS One. 2013;8(1), e53622.

14. Vivek N, Edward F, Steven B, Henry T, Jean J, Alan H. Early Bubble CPAP and outcomes in ELBW preterm infants. J Perinatol. 2003;23:195-9.

15. Kawaza K, Machen HE, Brown J, Mwanza Z, Iniguez S, Gest A, et al. Efficacy of a Low-Cost Bubble CPAP system in treatment of respiratory distress in a neonatal ward in Malawi. PLoS One. 2014;9(1), e86327.

16. Van Den Heuvel M, Blencowe H, Mittermayer K, Rylance S, Couperus A, Heikens $\mathrm{G}$, et al. Introduction of Bubble CPAP in a teaching hospital in Malawi. Ann Trop Paediatr. 2011;31:59-65. doi:10.1179/1465328110Y.0000000001.

17. Koyamaibole L, Kado J, Qovu JD, Colquhoun S, Duke T. An evaluation of bubble-CPAP in a neonatal unit in a developing country: effective respiratory support that can be applied by nurses. J Trop Pediatr. 2006;52(4):249-53.

18. Hedstrom A, Ryman T, Otai C, Nyonyintono J, McAdams RM, Lester D, et al. Demographics, clinical characteristics and neonatal outcomes in a rural Ugandan NICU. BMC Pregnancy Childbirth. 2014;14(1):327. doi:10.1186/1471-2393-14-327. 
19. Mwaniki MK, Gatakaa HW, Mturi FN, Chesaro CR, Chuma JM, Peshu NM, et al. An increase in the burden of neonatal admissions to a rural district hospital in Kenya over 19 years. BMC Public Health. 2010;10(1):591.

20. Liu L, Johnson HL, Cousens S, Perin J, Scott S, Lawn JE, et al. Global, regional, and national causes of child mortality: an updated systematic analysis for 2010 with time trends since 2000. Lancet. 2012;379(9832):2151-61.

21. Shrestha M, Basnet S, Shrestha PS. Bubble-CPAP in neonatal unit of TUTH. J Nepal Pediatr Soc. 2010;30(1):64-8.

Submit your next manuscript to BioMed Central and take full advantage of:

- Convenient online submission

- Thorough peer review

- No space constraints or color figure charges

- Immediate publication on acceptance

- Inclusion in PubMed, CAS, Scopus and Google Scholar

- Research which is freely available for redistribution 